\title{
Case Report \\ Central line-related bacteraemia due to Roseomonas mucosa in a neutropenic patient with acute myeloid leukaemia in Piraeus, Greece
}

\begin{abstract}
Correspondence
G. B. Christakis

chri-kis@hol.gr
\end{abstract}

Received 16 March 2006

Accepted 9 May 2006

\author{
G. B. Christakis, ${ }^{1}$ S. Perlorentzou, ${ }^{1}$ P. Alexaki, ${ }^{1}$ A. Megalakaki ${ }^{2}$ \\ and I. K. Zarkadis ${ }^{3}$ \\ 1,2Department of Microbiology ${ }^{1}$ and Haematology Clinic $^{2}$, METAXA Cancer Hospital,
18537 Piraeus, Greece
}

${ }^{3}$ Department of Biology, School of Medicine, University of Patras, 26500 Rion Patra, Greece

\begin{abstract}
A case of central venous catheter-related bacteraemia due to Roseomonas mucosa in a neutropenic patient with acute myelogenous leukaemia is reported. The patient was successfully treated with amikacin and piperacillin-tazobactam. The clinical isolate was identified as R. mucosa by $16 \mathrm{~S}$ rRNA gene sequencing.
\end{abstract}

\section{Introduction}

Roseomonas is a recently proposed genus of pink-pigmented, oxidative, short Gram-negative rods that includes five named species, Roseomonas gilardii (including Roseomonas gilardii subsp. rosea and Roseomonas gilardii subsp. gilardii), Roseomonas cervicalis, Roseomonas fauriae, Roseomonas mucosa and Roseomonas lacus, and three unnamed Roseomonas genomospecies, 4, 5 and 6 (Rihs et al., 1993; Han et al., 2003; Jiang et al., 2006). However, R. fauriae and genomospecies 6 have now been placed in the genus Azospirillum, which consists of plant growth-promoting bacteria (Cohen et al., 2004).

Although the natural reservoir of Roseomonas species remains unknown, isolates have been recovered occasionally from various environmental water sources (Rihs et al., 1993). Clinical isolates have been recovered from blood, wounds, exudates, abscesses, genitourinary sites, chronic ambulatory peritoneal dialysis fluid, corneal scrapings and bone (Nahass et al., 1995; Struthers et al., 1996; Sandoe et al., 1997; Tabin et al., 2001). However, according to Struthers et al. (1996), only $60 \%$ of isolates are associated with disease. Here, we describe a case of central venous catheter (CVC)-related bacteraemia caused by $R$. mucosa, in a neutropenic patient with acute myelogenous leukaemia.

\section{Case report}

A 39-year-old man with a prior history of M-4 acute myelogenous leukaemia was admitted to our hospital for chemotherapy. On day 93, during the third cycle of consolidation therapy (cytarabine, fludarabine), the patient

\footnotetext{
Abbreviation: CVC, central venous catheter.
}

The GenBank/EMBL/DDBJ accession number for the 16S rRNA gene sequence determined in this paper is AM161051. developed rigors and a temperature of $39 \cdot 5^{\circ} \mathrm{C}$. He had already been leukopenic $\left(0 \cdot 2 \times 10^{9} 1^{-1}\right)$ but had not been receiving any antibiotic prophylaxis. Laboratory investigation revealed a haematocrit of $25 \%, 8 \cdot 7 \mathrm{~g}$ haemoglobin $\mathrm{dl}^{-1}$; and a platelet count of $10 \times 10^{9} \mathrm{l}^{-1}$. All other biochemical laboratory tests were unremarkable. Chest X-ray as well as CT-scan of brain, chest and abdomen showed no signs of infection. Urine, stool and sputum cultures were negative. The site of the CVC, which had been placed at the time of admission, appeared abnormal with evidence of inflammation (erythema, swelling, tenderness).

During the episode, a short, Gram-negative, non-vacuolated rod, appearing in pairs or small chains (Fig. 1), was isolated from three blood samples drawn through the CVC (without quantification of colonies). Unfortunately, blood samples through the peripheral vein were not obtained. The CVC was removed and a culture of the Hickman catheter tip again showed the same Gram-negative coccobacillus. The patient was treated with amikacin ( $1 \mathrm{~g}$ per day) and piperacillintazobactam $(13.5 \mathrm{~g}$ per day) for 10 days. The patient became afebrile after the third day of therapy.

\section{Microbiological investigation}

Three sets of standard medium blood cultures in a total of six bottles were drawn within $24 \mathrm{~h}$. As mentioned above, the patient was not receiving any antimicrobial agent at the time of collection. Cultures were processed with the BacT/ALERT System (bioMeriéux). Bacterial growth was detected after $48 \mathrm{~h}$ incubation at $35^{\circ} \mathrm{C}$. Samples from three aerobic bottles grown on blood and chocolate agar formed pink, slightly mucoid colonies that did not autolyse after several days of incubation. The pink pigment, especially on chocolate agar, turned to red after 5 days of incubation. Growth also occurred at room temperature and at $42^{\circ} \mathrm{C}$, but not on 


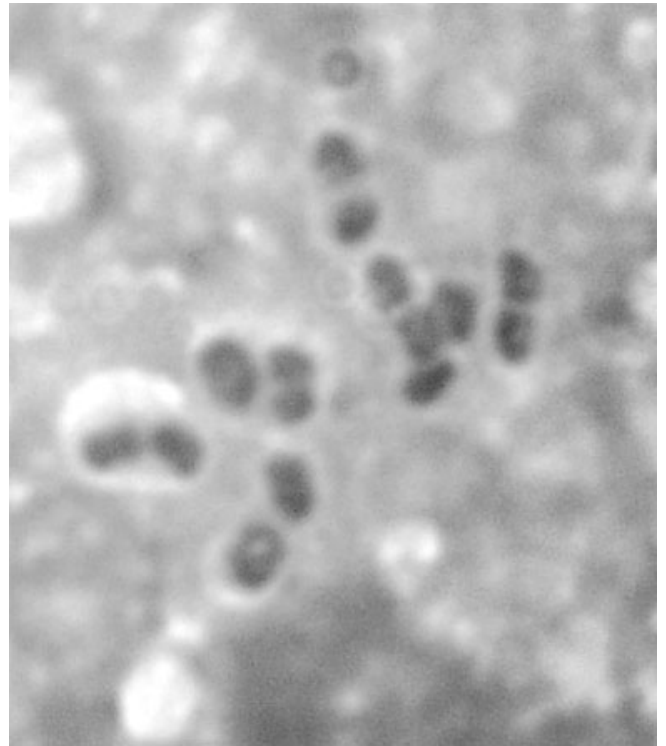

Fig. 1. Roseomonas mucosa: Gram stain of blood culture showing Gram-negative coccoid rods in pairs and small chains (magnification $\times 1000$ ).

MacConkey or anaerobic blood agar. The colonies were catalase-, oxidase- and urease-positive.

The isolate was identified as Roseomonas sp. (bionumber 054604, probability $99 \cdot 9 \%$ ) by using RapidID NF Plus (Remel Laboratories), whereas the API 20NE (bioMeriéux) computer database identified the organism as Methylobacterium mesophilicum (formerly named Pseudomonas mesophilica) (biotype 0201045, probability 97\%). The clinical isolate was further differentiated from M. mesophilicum on the basis of Gram-stain morphology (non-vacuolated rod), negative reactions for acid production from methanol and acetate utilization, and the inability to absorb long-wave UV light (Rihs et al., 1993).

In order to determine the primary structure of the $16 \mathrm{~S}$ rRNA gene of the clinical isolate, chromosomal bacterial DNA was isolated. Chromosomal DNA extraction, PCR and sequencing of the 16S rRNA gene were performed as described by Woo et al. (2000). The primers ROSEF1 (5'-ATCCTGGCTCAGAGCGAACG-3') and ROSER1 (5' CCCTACGGCTACCTTGTTACG-3') were used for PCR. The PCR product, $1447 \mathrm{bp}$ in size, was cloned in a pGEM-T plasmid vector (Promega) and sequenced using universal primers at least twice for both strands. The entire procedure was repeated and the PCR product was again sequenced. The nucleotide sequence of the PCR product (GenBank accession no. AM161051) was compared with known $16 \mathrm{~S}$ rRNA gene sequences by BLAST analysis. Genetic analysis confirmed that the isolate belongs to the genus Roseomonas, as the $16 \mathrm{~S}$ rRNA gene sequence was $93-99 \cdot 5 \%$ identical to those of Roseomonas spp. and 84-94\% identical with other members of Methylobacteriaceae. With regard to the species level, the highest identity score occurred with the strains 'Candidatus Roseomonas massiliae' 1461A (GenBank accession no. AF531769) and R. mucosa (AF538712). The 16S rRNA gene sequences of both were $99 \cdot 7 \%$ identical to that of the isolate, with four and five bases difference, respectively. In contrast, $99 \cdot 0 \%$ identity occurred with $R$. gilardii subsp. rosea (GenBank accession no. AY220740) and $R$. gilardii strain E9464 (AY150051), with 14 and 17 bases difference, respectively; the identity score with $R$. cervicalis and R. fauriae was $93 \%$.

Multiple alignment of $16 \mathrm{~S}$ rRNA gene sequences was performed with the CLUSTAL W program (Thompson et al., 1994) and phylogenetic trees were constructed using neighbour-joining, minimum evolution and parsimony analyses with MEGA version 3 software (Kumar et al., 2004). The isolate clustered with Roseomonas spp., as shown in Fig. 2. Bootstrap values of 99,99 and $90 \%$ in the neighbour-joining, minimum evolution and parsimony analyses, respectively, supported the branch separating Roseomonas spp. (including the isolate) from their closest relatives, Craurococcus roseus and Paracraurococcus ruber. More interestingly, the isolate was clearly different from the type strain of $R$. gilardii subsp. rosea and $R$. gilardii strain E9464 (data not shown), as well as from 'Candidatus Roseomonas massiliae', $R$. mucosa and $R$. gilardii ATCC $49956^{\mathrm{T}}$ with bootstrap values of 100,100 and $98 \%$ in the neighbour-joining, minimum evolution and parsimony analyses, respectively (Fig. 2).

Antimicrobial susceptibility testing of the isolate was carried out by using the panel Neg MIC Type 30 (MicroScan), according to the manufacturer's guidelines. MICs were read after 48 h incubation (Sandoe et al., 1997; Nolan \& Waites, 2005) using Clinical Laboratory Standards Institute interpretive criteria for non-fermentative Gram-negative bacteria (Clinical Laboratory Standards Institute, 2006). Escherichia coli ATCC 25922 and Pseudomonas aeruginosa ATCC 27853 were used as quality controls. The isolate was found to be sensitive to ciprofloxacin $\left(\leqslant 0.5 \mu \mathrm{g} \mathrm{ml}^{-1}\right)$, gentamicin, tobramycin, imipenem and meropenem $\left(\leqslant 1 \mu \mathrm{g} \mathrm{ml}^{-1}\right)$, levofloxacin and gatifloxacin $\left(\leqslant 2 \mu \mathrm{g} \mathrm{ml}^{-1}\right)$, tetracycline and amikacin $\left(\leqslant 4 \mu \mathrm{g} \mathrm{ml}^{-1}\right)$, cefepime $\left(4 \mu \mathrm{g} \mathrm{ml}^{-1}\right)$ and chloramphenicol $\left(\leqslant 8 \mu \mathrm{g} \mathrm{ml}^{-1}\right)$, and resistant to ceftazidime, aztreonam $\left(\geqslant 32 \mu \mathrm{g} \mathrm{ml}^{-1}\right)$, cefotaxime $\left(\geqslant 64 \mu \mathrm{g} \mathrm{ml}^{-1}\right)$, piperacillin-tazobactam $(\geqslant 128 /$ $\left.4 \mu \mathrm{g} \mathrm{ml}^{-1}\right)$, ticarcillin-clavulanic acid $\left(\geqslant 128 / 2 \mu \mathrm{g} \mathrm{ml}^{-1}\right)$ and trimethoprim-sulfamethoxazole $\left(\geqslant 4 / 76 \mu \mathrm{g} \mathrm{ml}^{-1}\right)$. Given that there are no published methodological guidelines or interpretive breakpoints for MICs of antimicrobial agents for Roseomonas spp. (Clinical Laboratory Standards Institute, 2006) and conflicting results regarding the sensitivity of Roseomonas isolates to $\beta$-lactams is not an uncommon finding (Rihs et al., 1993; Lewis et al., 1997; McLean et al., 2005), the MICs of $\beta$-lactams were confirmed by using Etest (AB Biodisk). With the exception of cefepime and carbapenems, the isolate was resistant to all $\beta$-lactams tested. 


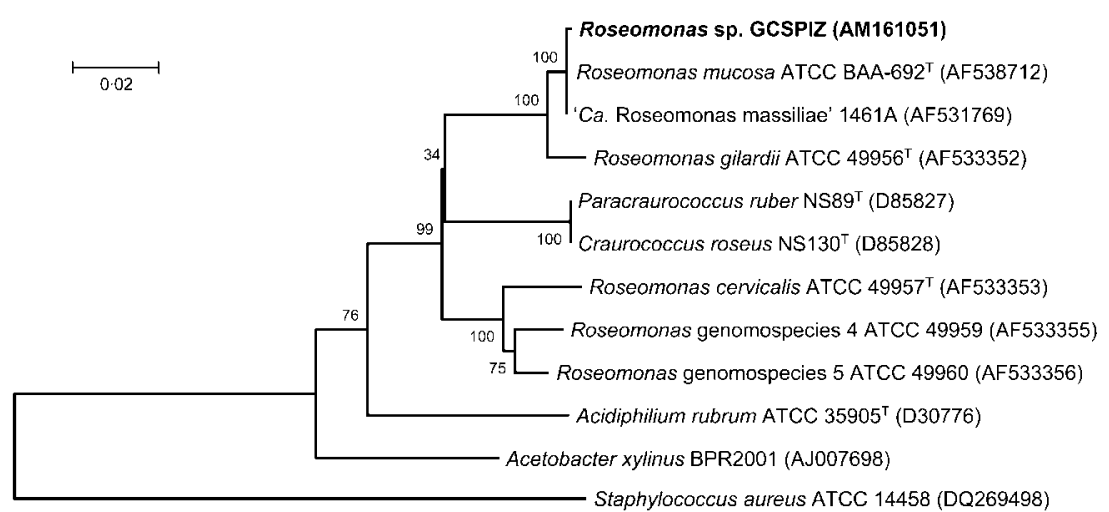

Fig. 2. Phylogenetic tree showing the relationship of the isolate (Roseomonas sp. GCSPIZ) with related sequences. The tree was constructed by using the neighbourjoining method, based on nearly complete sequences (1413 nt) of 16S rRNA genes. Bootstrap values resulting from 100 replications are given at branch points. Bar, 0.02 nucleotide changes per nucleotide position. Staphylococcus aureus was used as an outgroup.

\section{Discussion}

Enzymic activities and the ability to utilize various carbohydrates vary among the species and strains of Roseomonas. Therefore, definite identification based on acidification of various carbohydrates is difficult, inaccurate and timeconsuming. The RapidID NF Plus system identifies Roseomonas to the genus level with accuracy (Nolan \& Waites, 2005). Conversely, other commercial biochemical systems, such as API 20NE, can misidentify the organism (Schorch et al., 1999; Elshibly et al., 2005). Therefore, a sequencebased typing approach, such as analysis of $16 \mathrm{~S}$ rRNA genes, offers benefits for the identification of Roseomonas to the species level (Nolan \& Waites, 2005).

To our knowledge, 78 definite cases of Roseomonas bacteraemia have been reported in the literature. $R$. gilardii $(39 \cdot 7 \%)$ and R. mucosa $(29 \cdot 5 \%)$ were the two most common species (Schorch et al., 1999; Han et al., 2003; Dé et al., 2004; Elshibly et al., 2005; McLean et al., 2005). Given that only 37 clinical isolates have been characterized by using molecular methods (Han et al., 2003; Elshibly et al., 2005), the clinical and epidemiological significance of Roseomonas species remains relatively unknown. CVC-related bacteraemia is the most common clinical syndrome due to Roseomonas species, particularly in people with underlying malignancies or other chronic diseases (Dé et al., 2004). The production of biofilm on foreign materials may play an important role in the virulence of invasive infections due to species with a 'mucoid phenotype' (Elshibly et al., 2005). The outcome of Roseomonas CVC-related bacteraemia is favourable, even in patients with neutropenia. Therapy does not usually require removal of an indwelling device (Dé et al., 2004).

The choice of an effective drug for empirical treatment of infections due to Roseomonas spp. is sometimes difficult. According to the results of a recent review (Dé et al., 2004), the most active agents against Roseomonas species are amikacin and imipenem ( $\geqslant 99 \%$ susceptibility), followed by ciprofloxacin $(90 \%)$ and ticarcillin $(83 \%)$. Conversely, antibiotics such as third- or fourth-generation cephalosporins are not appropriate for treating infections due to this organism. Susceptibility varies among the different species;
$R$. mucosa has the highest risk of resistance whereas $R$. gilardii strains are the most susceptible (Han et al., 2003).

The 16S rRNA gene sequence of our isolate was $99 \cdot 7 \%$ identical to that of a strain provisionally named 'Candidatus Roseomonas massiliae' and a strain of R. mucosa (four and five bases difference, respectively). To date, 'Candidatus Roseomonas massiliae' has only been recovered by amoebal co-culture, from the nasal mucosa of a healthy homeless man (Greub et al., 2004). Our clinical isolate had remarkable phenotypic differences from $R$. mucosa strains studied by Han et al. (2003). (i) Colonies turned to red after several days of incubation, a phenotypic characteristic observed with strains of $R$. gilardii subsp. rosea and Roseomonas genomospecies 4 . In addition, the colonies were slightly mucoid and did not autolyse after several days of incubation. (ii) API $20 \mathrm{NE}$ reaction code matched with two (9\%) of the $22 R$. mucosa strains examined in case weakly positive reactions were excluded. (iii) The isolate was cefepimesensitive. It is worth noting that the patient discussed here was successfully treated with amikacin and piperacillintazobactam, in spite of the in vitro resistance of the clinical isolate to piperacillin-tazobactam.

In conclusion, our case underlines the clinical significance of Roseomonas species, particularly in the presence of an indwelling device. Differences in susceptibility patterns and virulence among the various species highlight the importance of definite identification of Roseomonas isolates. Broad-spectrum antibiotics (carbapenems and possibly quinolones) or combination therapy (including an aminoglycoside) is the first choice for the empirical treatment of Roseomonas infections. In contrast, consideration of coverage with a third- or fourth-generation cephalosporin would not be reasonable.

\section{References}

Clinical and Laboratory Standards Institute (2006). Performance standards for antimicrobial susceptibility testing, sixteenth information supplement, M100-S16. Wayne, PA: Clinical and Laboratory Standards Institute. 
Cohen, M. F., Han, X. Y. \& Mazzola, M. (2004). Molecular and physiological comparison of Azospirillum spp. isolated from Rhizoctonia solani mycelia, wheat rhizosphere, and human skin wounds. Can J Microbiol 50, 291-297.

Dé, I., Rolston, K. V. I. \& Han, X. Y. (2004). Clinical significance of Roseomonas species isolated from catheter and blood samples: analysis of 36 cases in patients with cancer. Clin Infect Dis 38, 1579-1584.

Elshibly, S., Xu, J., McClurg, R. B., Rooney, P. J., Millar, B. C., Alexander, H. D., Kettle, P. \& Moore, J. E. (2005). Central line-related bacteremia due to Roseomonas mucosa in a patient with diffuse large B-cell non-Hodgkin's lymphoma. Leuk Lymphoma 46, 611-614.

Greub, G., La Scola, B. \& Raoult, D. (2004). Amoebae-resisting bacteria isolated from human nasal swabs by amoebal coculture. Emerg Infect Dis 10, 470-477.

Han, X. Y., Pham, A. S., Tarrand, J. J., Rolston, K. V., Helsel, L. O. \& Levett, P. N. (2003). Bacteriologic characterization of 36 strains of Roseomonas species and proposal of Roseomonas mucosa sp. nov. and Roseomonas gilardii subsp. rosea subsp. nov. Am J Clin Pathol 120, 256-264.

Jiang, C.-Y., Dai, X., Wang, B.-J., Zhou, Y.-G. \& Liu, S.-J. (2006). Roseomonas lacus sp. nov., isolated from freshwater lake sediment. Int J Syst Evol Microbiol 56, 25-28.

Kumar, S., Tamura, K. \& Nei, M. (2004). MEGA3: integrated software for molecular evolutionary genetics analysis and sequence alignment. Brief Bioinform 5, 150-163.

Lewis, L., Stock, F., Williams, D., Weir, S. \& Gill, V. J. (1997). Infections with Roseomonas gilardii and review of characteristics used for biochemical identification and molecular typing. Am J Clin Pathol 108, 210-216.

McLean, T. W., Rouster-Stevens, K., Woods, C. R. \& Shetty, A. K. (2005). Catheter-related bacteremia due to Roseomonas species children with cancer. Pediatr Blood Cancer 44, 1-3.
Nahass, R. G., Wisneski, R., Herman, D. J., Hirsh, E. \& Goldblatt, K. (1995). Vertebral osteomyelitis due to Roseomonas species: case report and review of the evaluation of vertebral osteomyelitis. Clin Infect Dis 21, 1474-1476.

Nolan, J. S. \& Waites, K. B. (2005). Nosocomial ventriculitis due to Roseomonas gilardii complicating subarachnoid haemorrhage. J Infect 50, 244-251.

Rihs, J. D., Brenner, D. J., Weaver, R. E., Steigerwalt, A. G., Hollis, D. G. \& Yu, V. L. (1993). Roseomonas, a new genus associated with bacteremia and other human infections. J Clin Microbiol 31, 3275-3283.

Sandoe, J. A. T., Malnick, H. \& Loudon, K. W. (1997). A case of peritonitis caused by Roseomonas gilardii in a patient undergoing continuous ambulatory peritoneal dialysis. J Clin Microbiol 35, 2150-2152.

Schorch, P. E., Minniganti, V. R., Nair, R. \& Cunha, B. A. (1999). Primary Roseomonas gilardii bacteremia in a patient with inflammatory breast cancer. Clin Microbiol Newsl 21, 188-190.

Struthers, M., Wong, J. \& Janda, J. M. (1996). An initial appraisal of the clinical significance of Roseomonas species associated with human infections. Clin Infect Dis 23, 729-733.

Tabin, G., Danenhower, C., Reardon, D., Dimmig, J. \& McCormick, G. (2001). Opportunistic Roseomonas keratitis. Cornea 20, 772-773.

Thompson, J. D., Higgins, D. G. \& Gibson, T. J. (1994). CLUSTAL W: improving the sensitivity of progressive multiple sequence alignment through sequence weighting, position-specific gap penalties and weight matrix choice. Nucleic Acids Res 22, 4673-4680.

Woo, P. C. Y., Leung, P. K. L., Leung, K. W. \& Yuen, K. Y. (2000). Identification by $16 \mathrm{~S}$ ribosomal RNA gene sequencing of an Enterobacteriaceae species from a bone marrow transplant recipient. Mol Pathol 53, 211-215. 